\title{
A Novel Nonlinear BWR Stability Indicator Based on the Sample Entropy
}

\author{
Omar Alejandro Olvera-Guerrero (D), \\ Alfonso Prieto-Guerrero $\mathbb{D}$, and Gilberto Espinosa-Paredes
}

División de Ciencias Básicas e Ingeniería, Universidad Autónoma Metropolitana-Iztapalapa, Ciudad de México 09340, Mexico

Correspondence should be addressed to Alfonso Prieto-Guerrero; apg@xanum.uam.mx

Received 15 June 2018; Accepted 9 October 2018; Published 1 November 2018

Academic Editor: Alejandro Clausse

Copyright (c) 2018 Omar Alejandro Olvera-Guerrero et al. This is an open access article distributed under the Creative Commons Attribution License, which permits unrestricted use, distribution, and reproduction in any medium, provided the original work is properly cited.

\begin{abstract}
BWRs are thus far the simplest energy systems to transform fission energy into electrical power. However, there are still many aspects in their operation that, under certain conditions, may induce BWR unstable behavior. The default indicator to study BWR unstable behavior is the Decay Ratio (DR). However, due to the fact that BWRs show very complex responses under instability and responses that may even be chaotic, the DR might not be a suitable choice to rely on to accommodate for such intricate behavior. In this work a novel methodology based on the Sample entropy (SampEn) and the noise-assisted multivariate empirical mode decomposition (NA-MEMD) is introduced. Such methodology was developed thinking for a real time-implementation of a stability monitor. The proposed methodology was tested with a set of signals that stem from several nuclear power plants in operation today that have experienced in the past unstable events, each one of a different nature.
\end{abstract}

\section{Introduction}

The issue of BWR instability has been a topic of concern of many scientific and technological works for more than forty years. BWR unstable events are quite rare and may happen during BWR start up or during transients that may interfere with the conventional operating region of the reactor. BWR instability phenomena are strongly linked with the interaction between thermal hydraulic and neutron kinetics processes such interaction under certain conditions and may induce undesirable BWR behavior that could jeopardize BWR operation. Thus, a reliable prediction for the onset of BWR instability is of utter importance for BWR core safety [1].

D’Auria [2] presented all BWR unstable events recorded up to 1999 , which were due to various incidents (e.g., $[3,4])$, while others were intentionally induced for experimental purposes (e.g., $[5,6])$. Unstable oscillations in the neutron flux were recorded during these unstable events through the use of the electronic instrumentation available in the reactor. After the first events of instability were observed, the respective authorities fostered the growth of research projects to study the physical phenomena involved in BWR instability.
The most common instability type for commercial BWRs is the density-wave instability [7-9]. This type of instability can be described as follows: given a flow perturbation, a wave of voids travels upwards through the channel producing a pressure drop $\left(\Delta p=\Delta p_{1 \phi}+\Delta p_{2 \phi}\right.$, is approximately constant, where $\Delta p_{1 \phi}$ is the pressure drop in the single phase in the liquid region and $\Delta p_{2 \phi}$ is the pressure drop in the two-phase flow region) which is delayed with respect to the original perturbation. An increase in flow might induce an increase in pressure drop and a negative feedback that reduces the flow perturbation. The density wave phenomena delays this feedback and, at some frequency, the delay is equivalent to a $180^{\circ}$ phase lag; so at this frequency the pressure drop feedback is positive and if the gain is large enough, the channel flow becomes unstable and oscillates at that frequency highly concentrated around $0.5 \mathrm{~Hz}$, where the associated resonance frequency is related with the fluid transit time along the heated channel, which could differ from 2 seconds, i.e., approximately $1 \mathrm{~Hz}$.

In practice, the most commonly used indicator to study BWR stability due to wave oscillation is the decay ratio (DR), which is computed from the impulse response function 
obtained from a model fitting a linear second order system, accommodating the core behavior of a BWR. The DR is the current output indicator of most, if not all, of the stability monitors implemented in NPPs today [10]. The DR as a feasible BWR stability measure has been widely accepted but it has been observed that a BWR working at an operating point with a small DR can be close to instability [11]. Sometimes, the DR jumps from the stable to the far unstable region [12]. According to these works, the DR might not be a reliable option, under certain conditions. Besides, the need for linear and stationary signals might be a handicap for DR estimation. Therefore, it is necessary to explore new methodologies and indicators adapted to accommodate at their theoretical framework nonlinear and nonstationary behavior in order to study BWR unstable phenomena with as much realism as possible.

We are trying to convey in this work this idea, for this we explore the Sample Entropy (SampEn) linked to the noiseassisted multivariate empirical mode decomposition (NAMEMD) to infer whether BWR signals are stable or not. The SampEn [13] is a measure that provides an index of signal complexity or irregularity of a time series. In this way, the SampEn might work as a possible nonlinear BWR stability indicator. SampEn was in principle developed almost exclusively to analyze physiological time series [13] but its utility has expanded to other domains. For instance, SampEn has been tested with daily weather temperature to measure climate complexity [14]. It has been used to measure the complexity of the dynamic reconfiguration of the brain [15] to infer its association with normal aging.

To properly estimate the SampEn from real BWR signals, the NA-MEMD [16] was explored. The NA-MEMD is an algorithm that decomposes non-stationary signals that stem from nonlinear sources. This technique also mitigates the mode mixing phenomena [17] of the default EMD [18] technique. The EMD is the root technique that inspired the development of the NA-MEMD. The NA-MEMD produces a local and fully data-driven decomposition of a studied signal into its fast and slow oscillations. At the end of the procedure, the studied signal can be expressed as a sum of amplitude and frequency modulated (AM-FM) functions called intrinsic mode functions (IMFs), or simply called modes, plus a residue (the trend) of the decomposition. The combination of the NAMEMD and the Hilbert transform is known as the HilbertHuang transform (HHT). The methodology we introduce here is based on the HHT and it computes an indicator linked to BWR stability, in this case the aforementioned SampEn. The NA-MEMD decomposes the analyzed BWR signal into IMFs. One or more of these extracted modes can be associated to the instability problem in BWRs. Through HHT it is possible to get the instantaneous frequency (IF) linked to each IMF. By tracking this IF and the SampEn of the IMF linked to instability, the estimation of the SampEnbased stability indicator is accomplished. The methodology proposed in here is a continuation of two previous works $[19,20]$, in which a Shannon Entropy estimator [21] was used in conjunction with other members of the EMD family to study the stability of artificial and real BWR signals. In the present work the Shannon Entropy estimator was replaced by the SampEn practical formula to infer whether BWR signals are stable or not.

This work is organized as follows: in Section 2, a full review of the SampEn and of the NA-MEMD techniques is given. In Section 3, the methodology to compute the instantaneous frequency and the proposed SampEn novel stability indicator is described. In Section 4 , the validation of the methodology presented in this paper is performed doing experiments with real signals taken from the Forsmark [5] and Ringhals [6] stability benchmarks and from a typical BWR power plant during stable and unstable operation. Our major observations regarding our novel methodology based on SampEn and the NA-MEMD are thoroughly discussed in Section 5.

\section{Preliminaries}

2.1. The Sample Entropy. The procedure for computing SampEn was introduced by Richman and Moorman [13]. In this work, we grant a brief summary of their findings. SampEn is a measure of complexity. Let $x=\left[\mathrm{x}_{1}, \mathrm{x}_{2}, \ldots, \mathrm{x}_{N}\right]$ be a time series of length $N$. To compute the complexity of this time series via SampEn, follow the next steps:

(1) Build a vector $v_{i}$ with $m$ consecutive data points taken from $x$

$$
v_{i}=\left[\mathrm{x}_{i}, \mathrm{x}_{i+1}, \ldots, \mathrm{x}_{i+m-1}\right]
$$

where $m$ is the length of sequences to be compared, also called the embedding dimension.

(2) For each $i$ define

$$
C_{i}^{m}=\frac{1}{N-m-1} \sum_{j=1, j \neq i}^{N-m} \Theta\left(r-\left\|v_{i}-v_{j}\right\|_{1}\right)
$$

where $i$ varies in the interval $(1 \leq \mathrm{i} \leq \mathrm{N}-m)$. In here, $r$ is the tolerance for accepting matches, $r=$ $s \times \operatorname{std}(x)$, where $s$ is a scaling parameter and $\operatorname{std}(x)$ is the standard deviation of $x . \Theta(\cdot)$ is the Heaviside function:

$$
\Theta(\mathrm{x})= \begin{cases}0, & x<0 \\ 1, & x \geq 0\end{cases}
$$

and $\|\cdot\|_{1}$ is the Chebyshev distance, defined as

$$
\begin{aligned}
& \left\|v_{i}-v_{j}\right\|_{1} \\
& \quad=\max \left(\left|x_{i}-x_{j}\right|,\left|x_{i+1}-x_{j+1}\right|, \ldots,\left|x_{i+m-1}-x_{j+m-1}\right|\right)
\end{aligned}
$$

(3) $C_{i}^{m}$ represents the proportion of $v_{j}(i \neq j)$ whose distances to $v_{i}$ are less than $r$. Now, for each $i$ we also define

$$
C_{i}^{m+1}=\frac{1}{N-m-1} \sum_{j=1, j \neq i}^{N-m} \Theta\left(r-\left\|v_{i}-v_{j}\right\|_{1}\right)
$$


where $C_{i}^{m+1}$ represents the proportion corresponding to the dimension of $m+1 . C_{i}^{m}$ and $C_{i}^{m+1}$ have the same mold, but embedding vectors in both cases are defined in different spaces.

(4) Average across all embedding vectors, to obtain

$$
U^{m}=\frac{1}{N-m} \sum_{i=1}^{N-m} C_{i}^{m}
$$

and

$$
U^{m+1}=\frac{1}{N-m} \sum_{i=1}^{N-m} C_{i}^{m+1}
$$

(5) The SampEn is computed as

$$
\text { SampEn }=-\ln \left(\frac{U^{m+1}}{U^{m}}\right)
$$

SampEn is the negative natural logarithm of the conditional probability that two sequences similar for $m$ points remain similar at the next point, where self matches are not included in calculating the probability. Thus, a lower value of SampEn indicates more self-similarity (i.e., high order) of the studied time series whereas a higher value of SampEn points to higher complexity of the time series.

The calculation of SampEn requires a priori determination of two unknown parameters, $m$ and $r$ (the length of data $N$ is up to the user). The suggested values of $r$ are located in the range of 0.1-0.2 times the standard deviation of the studied signal $x$. In this work, we looked for the $r$ value that grants the global SampEn maximum because this maximum value leads to the correct interpretation of signals complexity [22]. So, this $r$ of interest is fixed in our simulations at $r=0.1$. The value of $m$ can be computed via the estimation of false nearest neighbor [23]. In our particular BWR stability discipline, $m$ that was found through false nearest neighbors for our signals was most of the time equal to 2 . Therefore, $m$ is fixed at $m=2$ for all of our computations. SampEn is, theoretically speaking, a fraction in the interval $0<$ SampEn $<\infty$. However, the next two formulas can be used to find the lower bound and the upper bound of SampEn for fixed values of $m$ and $N$.

The lower bound is computed as

$$
2[(N-m-1)(N-m)]^{-1}
$$

The upper bound is computed as

$$
\ln (N-m)+\ln (N-m-1)-\ln (2)
$$

For all of our simulations $m, r$ and $N$ are fixed at $m=2$, $r=0.1 \times \operatorname{std}(x)$ and $N=300$ data points. Thus the lower bound is practically $\approx 0$ whereas the upper bound is close to 11. However, the upper bound value was never attained for any simulation.

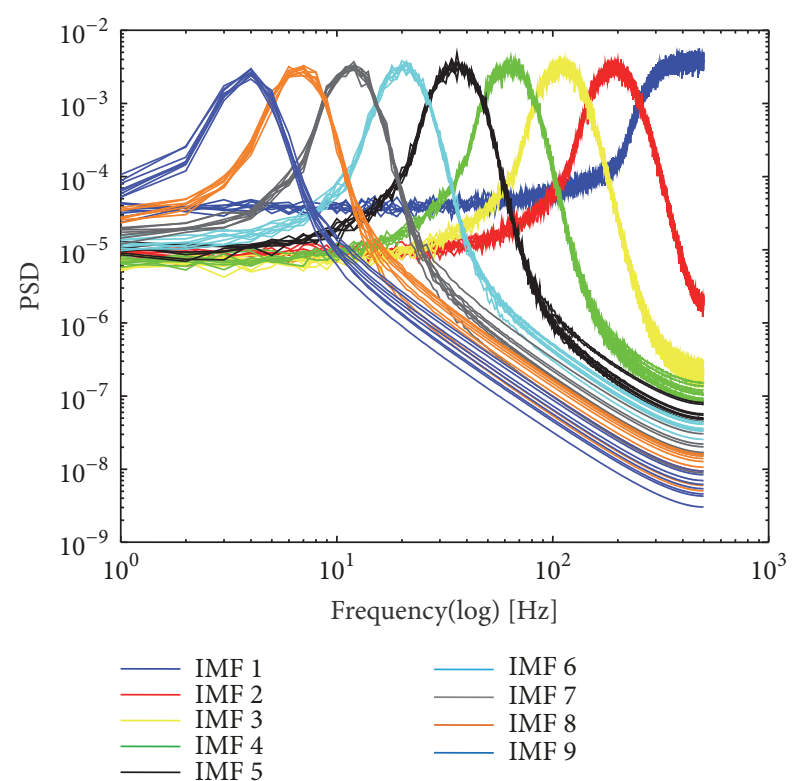

FIGURE 1: Averaged spectra of IMFs (1-9) obtained from 50 realizations of eight-channel white Gaussian noise via MEMD. Reproduced from [20].

2.2. The Noise-Assisted Multivariate Empirical Mode Decomposition. The multivariate empirical mode decomposition [16], commonly referred to as the MEMD, is a nonlinear filter to make the classic EMD [18] suitable for processing of multichannel signals. The behavior of the MEMD was analyzed in the presence of white Gaussian noise $[20,24]$ and it was found that the MEMD in essence acts as a dyadic filter bank on each channel of the multivariate input signal; such MEMD property is illustrated in Figure 1 and its steps are given below.

The MEMD has the property of aligning the IMFs or modes from different channels across the same frequency range and the NA-MEMD technique was developed to aid resolve the mode mixing problem observed in the current MEMD, two key issues for real applications. The NA-MEMD technique, which makes use of the quasi-dyadic filter bank properties of MEMD on white noise (Figure 1), is capable of reducing the mode mixing problem for classes of signals where the quasidyadic filter bank structure proves useful (such property applies for BWR signals). The NAMEMD adds $q$ extra channels of white noise to the input studied signal before being decomposed by the MEMD. The addition of these $q$ extra channels helps to establish a uniformly distributed reference scale which, in turn, results in corresponding modes exhibiting a quasi-dyadic filter bank structure. The extracted IMFs corresponding to the $q$ extra channels of white noise are then discarded yielding a set of IMFs linked with only the original input signal of interest. The NA-MEMD procedure is summarized in the following next steps [20]:

Step 1. Create an uncorrelated Gaussian white noise timeseries ( $q$-channels) of the same length as that of the input. 
Step 2. Add the noise channels ( $q$-channels) created in Step 1 to the input multivariate ( $L$-channels) signal, obtaining an $(L+q)$-channel signal.

Step 3. Process the resulting $(L+q)$-channel multivariate signal using the MEMD [16] algorithm, to obtain multivariate IMFs.

Step 4. From the resulting $(L+q)$-variate IMFs, discard the $q$ channels corresponding to the noise, giving a set of $L$-channel IMFs corresponding to the original signal.

However, it should be mentioned that the NA-MEMD for mitigating the mode mixing becomes more useful for signals in which the dyadic filter bank decomposition is relevant. This is the case for the studied BWR signals.

\section{BWR Stability Methodology Based on the SampEn and the NA-MEMD}

The methodology to detect stable or unstable states (this methodology works with raw data. So, there is no need to preprocess the analyzed data) in a core of a BWR, based on the Sample entropy and the NA-MEMD, is presented in the next steps:

(1) The studied raw signal (APRM or LPRM) obtained from the BWR is segmented in windows of $60 \mathrm{~s}$ of duration.

(2) Each segment (of $60 \mathrm{~s}$ of time span) is considered as just an independent channel $(L=1)$ added with $q$ independent channels of white Gaussian noise and decomposed through the NA-MEMD (for all of our computer simulations $q=2$ ).

(3) After decomposition, the $q$ channels corresponding to the noise are discarded, giving a set of IMFs corresponding to each decomposed studied segment.

(4) The Hilbert transform of each IMF (i.e., the HHT) is computed to get the instantaneous frequency of each IMF in the studied segment.

(5) When tracking these frequencies, it is possible to get the mode (or modes) linked to the density wave oscillation. In this way, only the mode (or modes) associated to BWR instability is considered for further processing whereas the remaining extracted modes are ignored.

(6) The SampEn of the tracked mode of interest (IMF) is computed for $N=300$ (the length of the analyzed segment of $60 \mathrm{~s}$ ), $m=2$ and $r=0.1 \times \operatorname{std}($ segment) to attain the global maximum of the SampEn that leads to the correct interpretation of the studied segment complexity [22].

(7) The mean, median, and the variance of the SampEn values are computed and averaged along all the studied segments of $60 \mathrm{~s}$ of the original signal.

A high SampEn estimate $(>1)$ points out high irregularity and high unpredictability of the studied BWR signal (LPRM or APRM) and this high value might indicate that the studied signal could be merely broad band non-coherent noise (BBNCN). This BBNCN, from the BWR point of view is a sign of stable behavior. On the other hand, a low SampEn value $(<0.8)$ points to high predictability and regularity of the studied BWR signal. Such low value might indicate that the studied signal could be a cyclic one. Such waveform is a sign of BWR unstable behavior, due to the presence of a density wave that manifests in the LPRM or APRM signals as a cyclic waveform with a natural frequency that oscillates very close to $0.5 \mathrm{~Hz}$. For forced convection phenomena, in the circulation natural convection the effect of the chimney changes this value $[8,9]$.

\section{Results and Discussions}

Now we validate the methodology given in Section 3 with the next data sets, where $f_{s}$ is the sampling frequency of each set:

(1) Data set from a typical BWR:

(a) 208 steady state condition signals (i.e. stable signals), $f_{s}=5 \mathrm{~Hz}$.

(b) 96 unstable signals, $f_{s}=10 \mathrm{~Hz}$.

(2) Case 4 of the Forsmark stability benchmark [5]:

(a) 1 APRM signal plus 22 LPRM signals, $f_{s}=12.5$ $\mathrm{Hz}$.

(3) Case 9 cycle 14 of the Ringhals stability benchmark [6]:

(a) 72 LPRM signals (located within 2 levels of the Ringhals reactor, each level has a total of 36 LPRMs, such levels are called 2 and 4$), f_{s}=12.5$ $\mathrm{Hz}$.

4.1. Stable Signals. The aim of this test is to observe the demeanor of the BWR in steady-state conditions. The results obtained are presented in Figures 2-8. Figure 2 shows the plot of one APRM stable signal of a typical BWR. Figure 3 shows in red dotted line, a studied segment of $60 \mathrm{~s}$ of duration of the APRM signal with its power spectral density (PSD) estimate (bottom of figure). Figure 4 shows the first four extracted IMFs of the studied signal, which happens to be noise that is decomposed in a quasidyadic fashion by the NA-MEMD. Figure 5 shows the power spectral density (PSDs) of some IMFs.

Figure 6 shows the tracked IMF (IMF 3 ) of interest whose instantaneous frequency (IF) oscillates around $0.5 \mathrm{~Hz}$. The computed SampEn of this IMF is 0.9351. Such high SampEn value indicates that the studied signal is BBNCN, so the studied segment is stable and far from an unstable behavior. In this stable case, a mode exist whose IF is close to $0.5 \mathrm{~Hz}$. However, the IMF 3 time series looks distant from a cyclic function we look for when an unstable event triggers in the core. All of the IFs were computed through the HilbertHuang transform of the extracted IMFs. Figure 7 shows the computed SampEn along time for all of the studied segments 


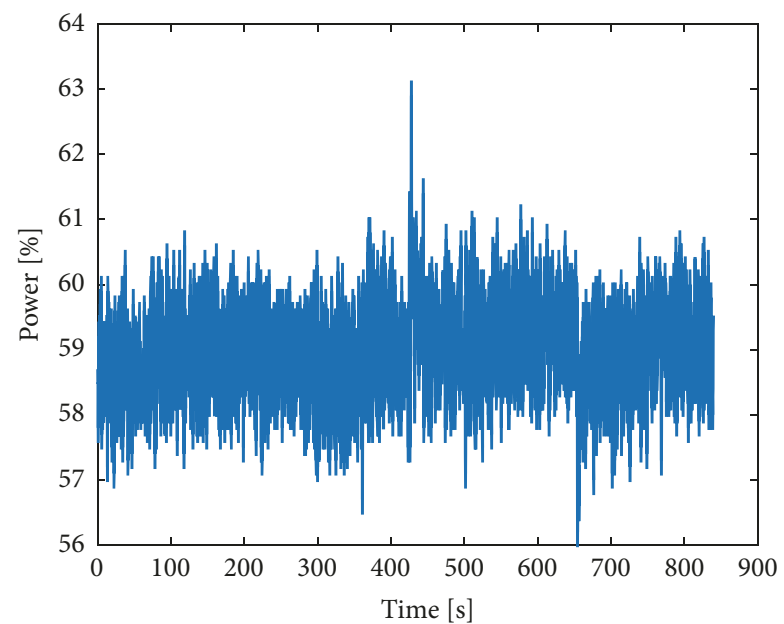

Figure 2: Stable signal from a typical BWR.
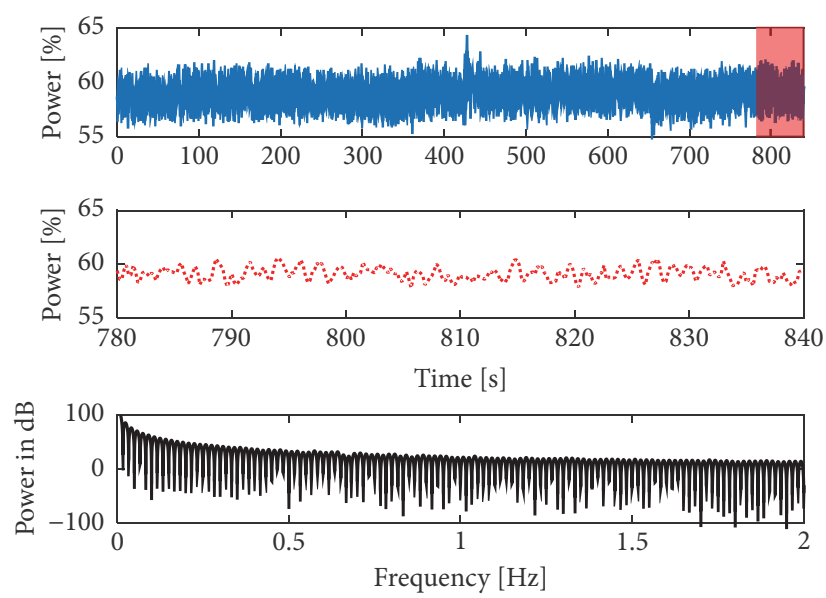

Figure 3: Analyzed segment of the studied APRM signal.
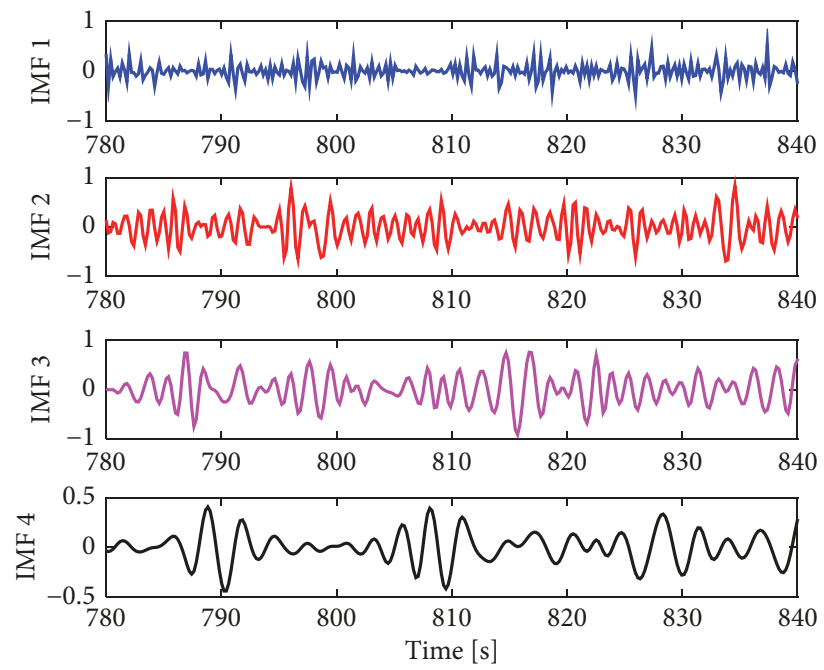

Figure 4: A plot of the first 4 extracted IMFs in the analyzed segment.
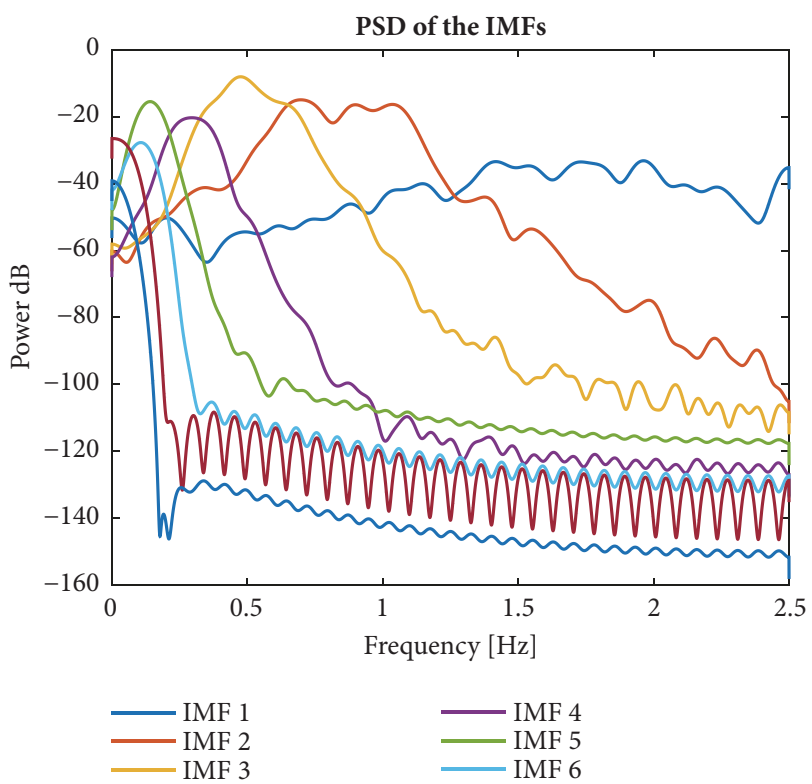

FIgURE 5: PSD estimate of the first 6 extracted IMFs in the analyzed segment.
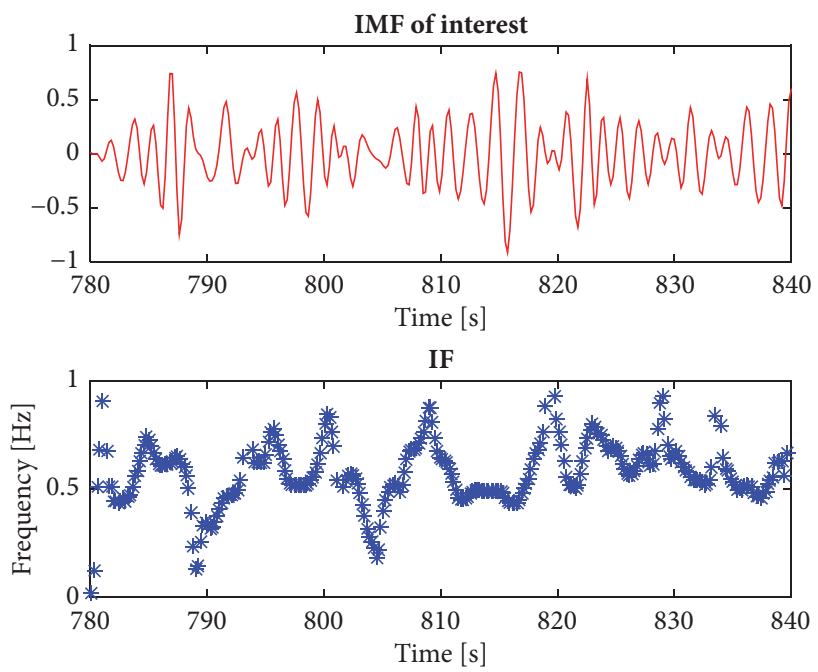

FIgURE 6: Tracked IMF (IMF 3) of interest and its associated instantaneous frequency (IF) around $0.5 \mathrm{~Hz}$ computed through the Hilbert-Huang transform.

TABle 1: Mean(SampEn), Median(SampEn), and std(Sampen) of the analyzed APRM.

\begin{tabular}{lccc}
\hline Signal & Mean(SampEn) & Median(SampEn $)$ & Std(SampEn) \\
\hline APRM & 1.1288 & 1.0994 & 0.1398 \\
\hline
\end{tabular}

of $60 \mathrm{~s}$; most of the SampEn estimates have a value slightly greater than 1 (i.e., the studied signal is stable). Table 1 shows the mean(SampEn), median(SampEn) and std(SampEn) of the time series shown in Figure 7.

Figure 8 shows a plot of the computed mean SampEn estimates of the 208 signals (each SampEn estimate is a blue point). A pattern appears, all of the mean SampEn 


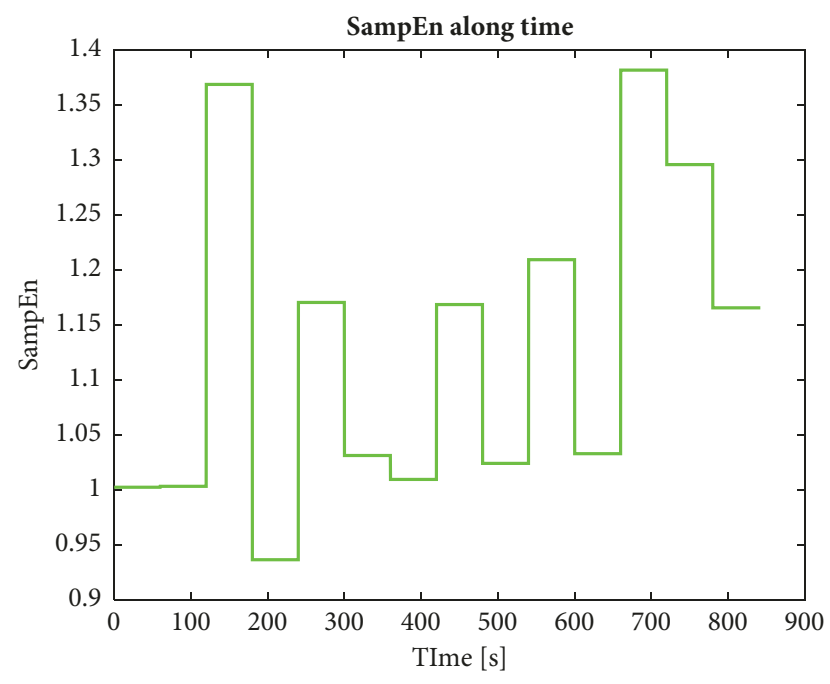

FIGURE 7: SampEn along time for the studied APRM stable signal.

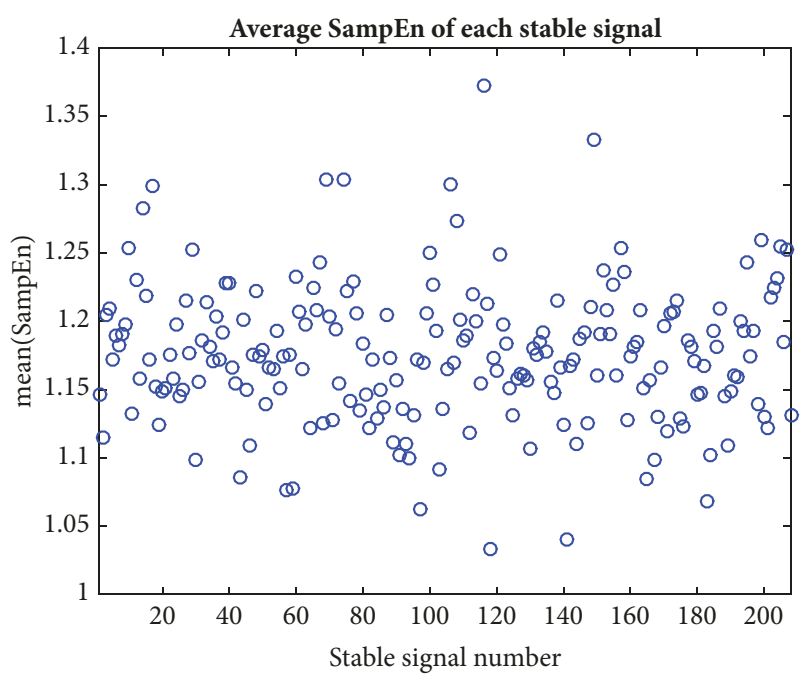

FIgUre 8: Computed mean(SampEn) of the studied 208 stable signals.

estimates for the studied signals are higher than 1 . Thus, the mean SampEn values point to BBNCN. Through SampEn estimation, the proposed stability methodology is able to detect stable signals and classify them in the stable category if their SampEn is greater than 1 .

4.2. Unstable Signals. The studied signal in this subsection stems from an instability event. This instability case is registered by 96 LPRM signals. For reasons of space, only the analysis of one signal is presented. Figure 9 shows the plot of the studied signal (from LPRM 2); this signal looks like a cyclic function. Figure 10 shows a studied segment of 60 s (red dotted line) and its PSD estimate (bottom of figure), the segment looks like a noisy cyclic function. Figure 11 shows the first 4 extracted IMFs of the studied segment;

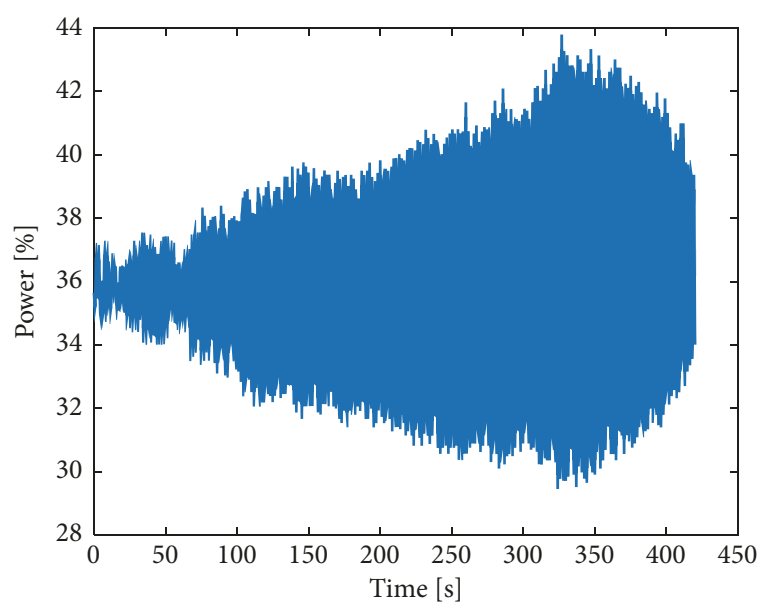

FIGURE 9: Unstable signal from a typical BWR.
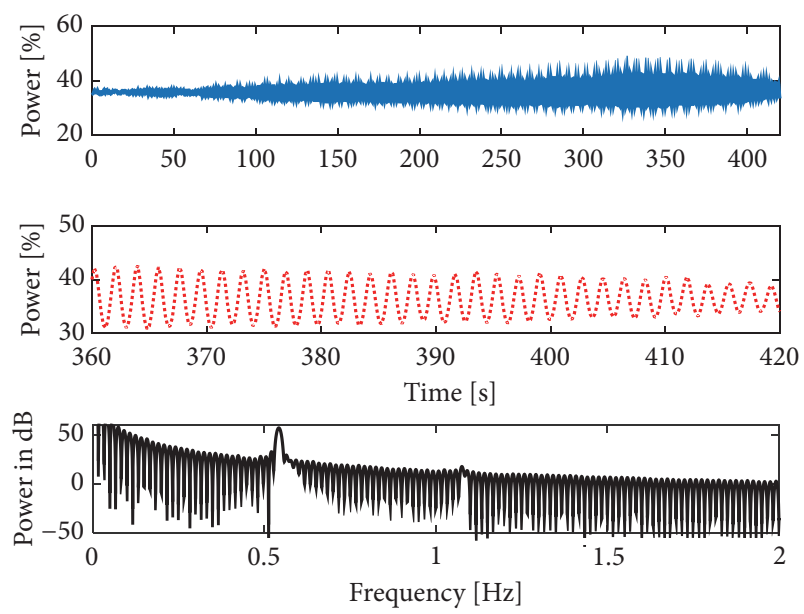

FIgURE 10: Analyzed segment of the studied LPRM 2 signal.
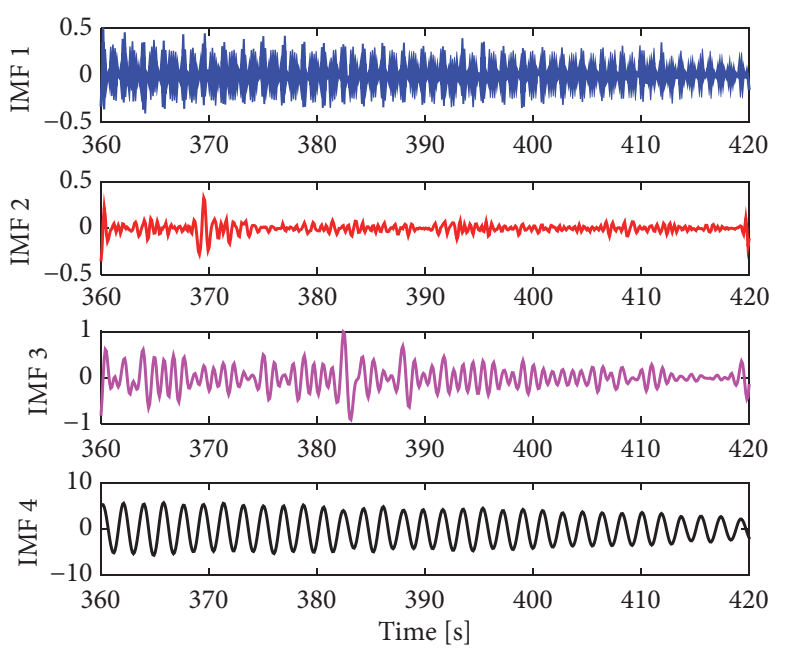

FIgURE 11: A plot of the first extracted 4 IMFs in the analyzed segment. 
TABLE 2: Mean(SampEn), Median(SampEn), and std(Sampen) of the analyzed LPRM 2. Now the mean(IF) and std(IF) are included.

\begin{tabular}{lccccc}
\hline Signal & Mean(SampEn) & Median(SampEn) & Std(SampEn) & Mean(IF) $[\mathrm{Hz}]$ & Std(IF) $[\mathrm{Hz}]$ \\
\hline LPRM 2 & 0.5425 & 0.5258 & 0.0544 & 0.5374 & 0.0030 \\
\hline
\end{tabular}

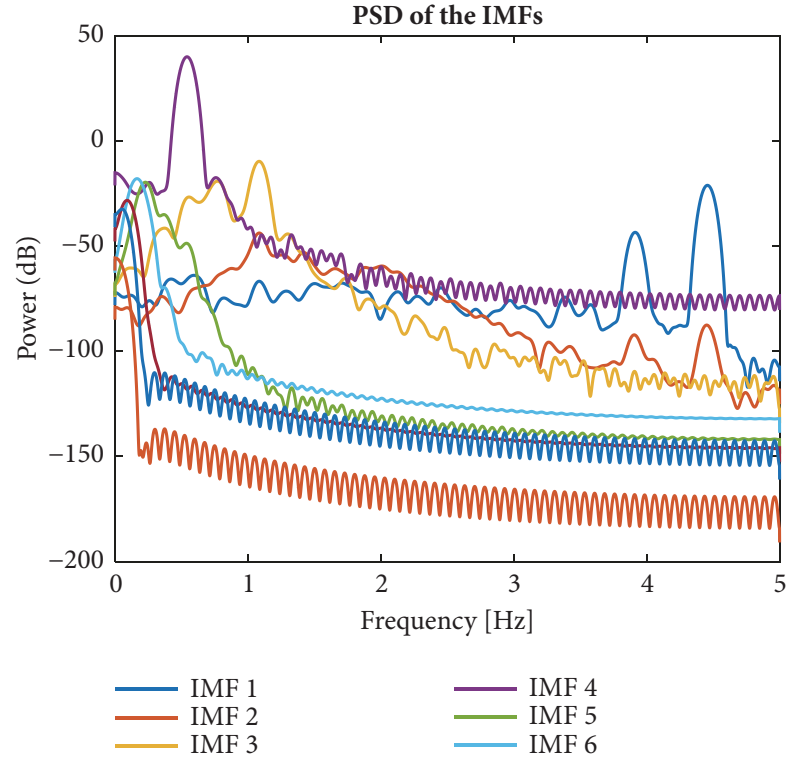

FIGURE 12: PSD of the first 6 extracted IMFs in the analyzed segment. The PSD of the IMF 4 is highly concentrated around $0.5 \mathrm{~Hz}$.
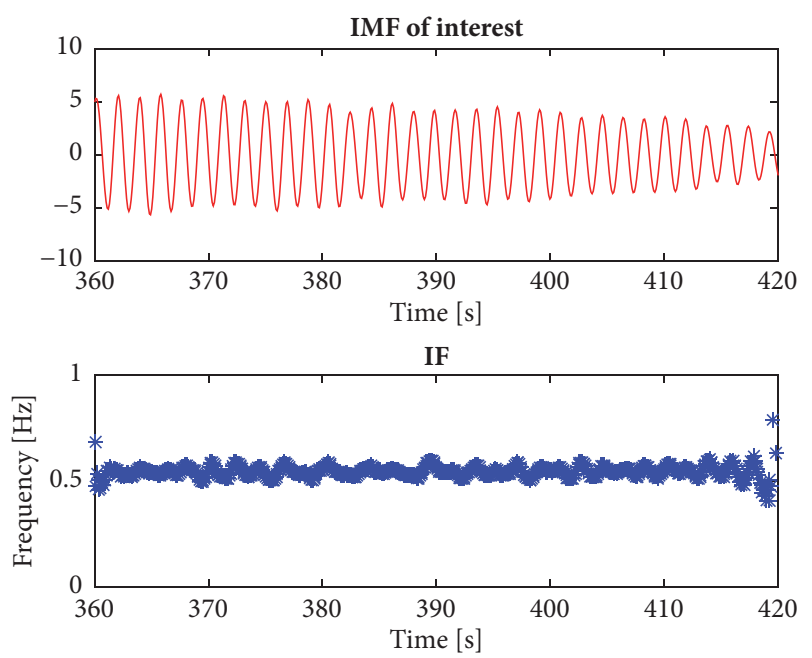

FIGURE 13: Tracked IMF (IMF 4) of interest linked to BWR instability and its associated instantaneous frequency (IF) around $0.5 \mathrm{~Hz}$ computed through the HHT.

the first 3 IMFs are linked with acquisition noise, whereas IMF 4 looks like a cyclic function (this is in fact the type of waveform commonly associated with density wave unstable events). Figure 12 shows the PSD estimates of the first 6 IMFs, the energetic content of IMF 4 is highly concentrated around $0.5 \mathrm{~Hz}$, and the energetic content of the other IMFs is meaningless next to the energy of IMF 4 . Figure 13 shows the time domain series of IMF 4 (which happens to be the mode associated to BWR instability) and its associated IF

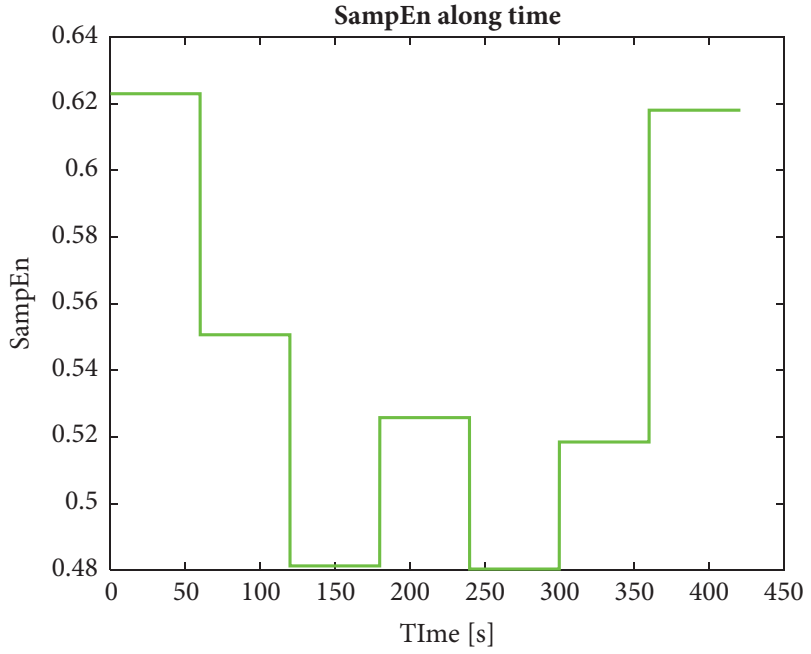

FIGURE 14: SampEn estimates of the IMFs or modes of interest along time.

that oscillates around $0.5 \mathrm{~Hz}$. The IF was computed via HHT. Now, this IF (IF 4) carries physical meaning [7]. The SampEn estimate of IMF 4 (IMF or mode linked to instability, see IF in Figure 13 and PSD estimate in Figure 12) is 0.6181; this estimate is a clear indication of signal regularity and low complexity. The SampEn points to a simple function; in this case a cyclic function that in our context is linked with a density wave type of instability. Thus, the studied segment is unstable. Figure 14 shows the SampEn estimates along time for all of the studied segments of the signal of interest; all estimates are smaller than 1.

Table 2 shows the mean(SampEn), median(SampEn), and std(SampEn) of the time series shown in Figure 14. Now, the tracked IF (IF 4) along time carries physical meaning. Thus, the mean(IF) and std(IF) values of all of the studied segments are now provided in this table.

Figure 15 shows a plot of the computed mean SampEn estimates of the 96 unstable signals (each SampEn estimate is a red point). A pattern appears, all of the mean SampEn estimates for the studied signals are smaller than 0.8. Thus, the mean SampEn values point to visually regular signals such as cyclic functions. Through SampEn estimation, we are able to build a binary classifier that separates BBNCN signals (linked to stability) from cyclic ones (linked to density wave instability). One core feature of SampEn is that it accommodates for complex nonlinear and nonstationary data whereas conventional Decay Ratio estimates must assume beforehand that the studied BWR signals behave linearly (an assumption that is false in real life).

4.3. Comparison between Stable and Unstable States. To complete the analysis of the stable/unstable signals through SampEn, Figure 16 shows a comparison of the mean SampEn 


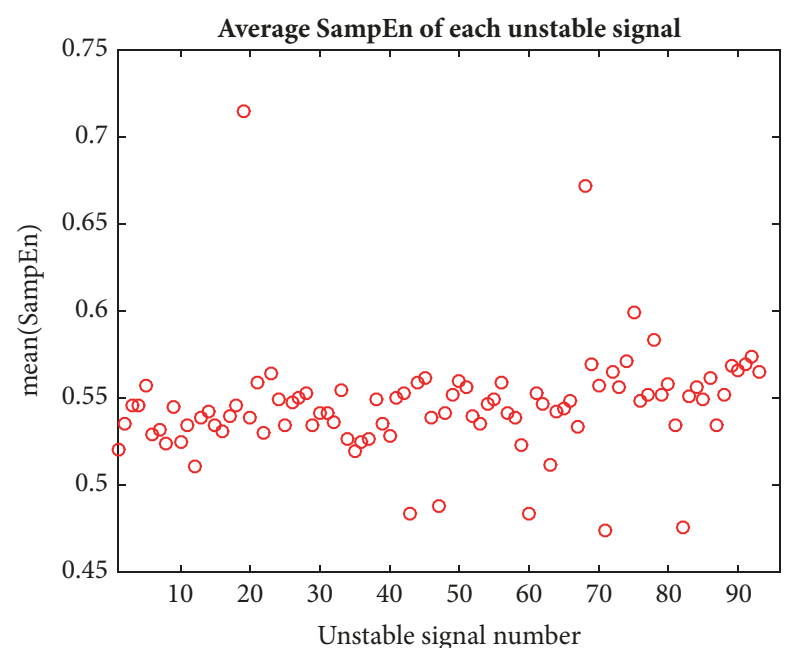

Figure 15: Computed mean(SampEn) of the studied 96 unstable signals.

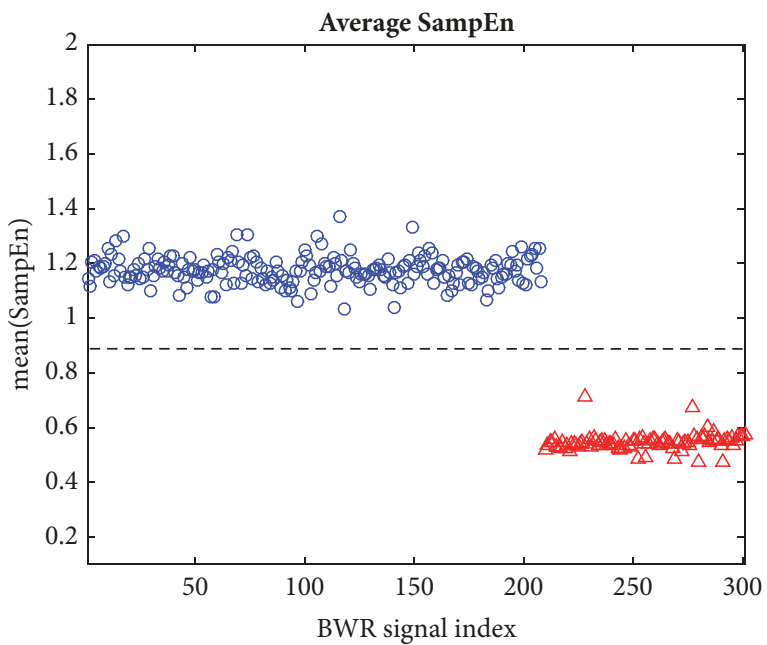

- Stable signals

$\triangle$ Unstable signals

FIgURE 16: Comparison of the mean(SampEn) values for both studied sets (a stable set of 208 APRM signals versus an unstable set of 96 LPRM signals).

values of the studied sets (stable set of 208 signals plus a set of 96 unstable signals). The computed SampEn mean values of the stable signals fluctuate very close to 1.2 whereas the SampEn mean values of the unstable signals oscillate very close to 0.6. So the SampEn detects with success stable BWR signals from unstable ones via complexity analysis (of course with the aid of the NA-MEMD to denoise these signals and isolate the mode linked to instability). There is a distance of $\approx$ 0.6 between the two states (stable from unstable). So, by fixing a threshold value around 0.9 , it is possible to differentiate one state from the other properly. Any SampEn estimate of a BWR signal segment higher than 0.9 is stable whereas any lower SampEn value from this threshold is unstable.

4.4. Case 4 of the Forsmark Stability Benchmark. Case 4 of the Forsmark stability benchmark [5]. This event is considered a

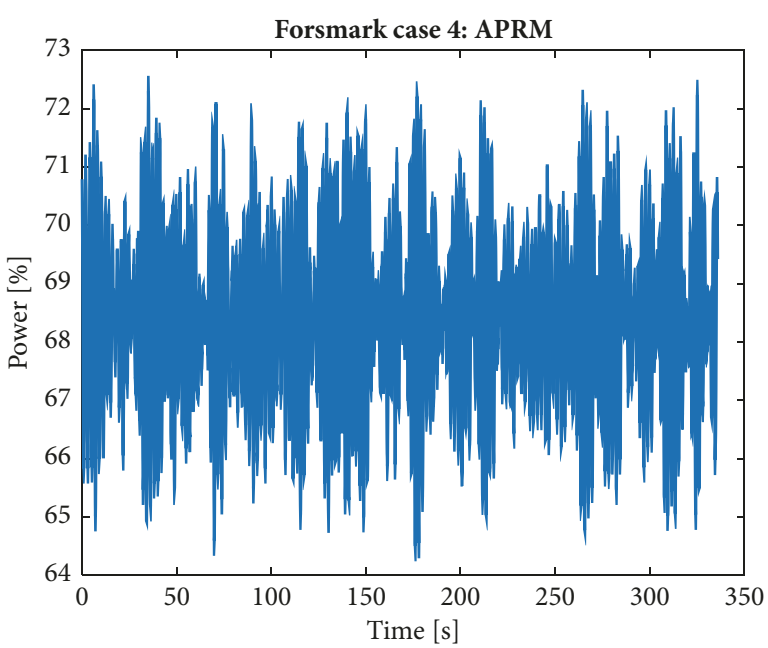

FIGURE 17: Forsmark Case 4 APRM signal.

difficult case to study by the complexity of the phenomena. For reasons of space, only the analysis of one case will be presented in detail. This case contains a mixture between a global oscillation and a regional oscillation. This particular event corresponds to a situation where the neutronic power reactor suffers unusual unstable problems, i.e., presenting a mix of oscillation modes. The C4_APRM and C4_LPRM_x signals correspond to average power monitors (APRM) and local range monitors LPRM. The entire case 4 consists of a total of 23 signals, 22 LPRMs and 1 APRM.

Nonetheless, only the analysis of the APRM is presented in this work. Figure 17 shows the studied APRM signal of interest. Figure 18 shows a studied $60 \mathrm{~s}$ seconds segment (the dotted red line) that is decomposed through NA-MEMD and its PSD estimate (bottom of figure). Figure 19 shows the first 5 extracted IMFs of the studied segment (as a reminder, the IMFs of the 2 noise extra channels are discarded). Figure 20 shows the PSD estimates of the first 6 IMFs; we highlight that there is a bit of mixing between IMF 4 and IMF 5 . Nonetheless IMF 5 is slightly closer to $0.5 \mathrm{~Hz}$ than the IMF 4 and has more energy. Figure 21 shows a plot of the IMF 5 of interest that is linked to BWR density wave instability and its associated instantaneous frequency (IF) that oscillates around to $0.5 \mathrm{~Hz}$ (we highlight that IMF 5 is slightly mixed with IMF 4 but this is a result due to the complexity of the Forsmark Case 4. Nevertheless, IMF 5 has more energy than IMF 4). The SampEn of this IMF 5 is 0.5443 . Thus this segment is unstable (SampEn points to high regularity and low complexity). Figure 22 shows the SampEn estimates of the IMFs or modes of interest along time for all of the studied segments of $60 \mathrm{~s}$. All of the SampEn estimates are smaller than 0.7. So, the studied APRM signal is unstable.

Table 3 shows the mean(SampEn), median(SampEn), and std(SampEn) of the time series shown in Figure 22 (SampEn estimates of IMF 4). The mean(IF) and std(IF) are also given in this table. Figure 23 shows a plot of the computed mean SampEn estimates of the 23 unstable signals (each SampEn estimate is a red point). A pattern appears; all of the mean SampEn estimates for the studied signals are 
TABle 3: Mean(SampEn), Median(SampEn), and std(Sampen) of the analyzed APRM. Now the mean(IF) and std(IF) are included.

\begin{tabular}{lcccc}
\hline Signal & Mean(SampEn) & Median(SampEn) & Std(SampEn) & $\begin{array}{c}\text { Mean(IF) } \\
{[\mathrm{Hz}]}\end{array}$ \\
\hline Forsmark APRM & 0.5369 & 0.5295 & 0.0411 & $\begin{array}{c}\text { std(IF) } \\
{[\mathrm{Hz}]}\end{array}$ \\
\hline
\end{tabular}
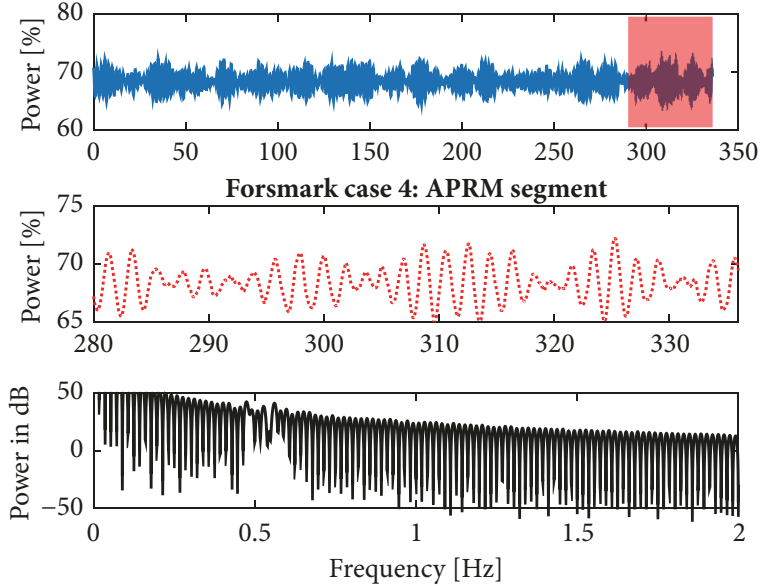

FIGURE 18: Studied segment of the Forsmark Case 4 APRM.
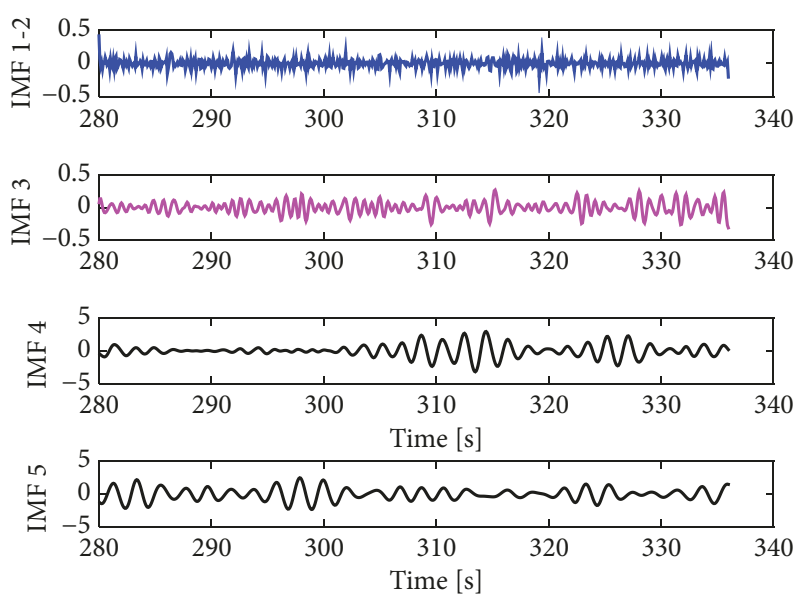

FIGURE 19: A plot of the first 5 IMFs in a segment of the studied Forsmark APRM.

smaller than 0.8. Thus, the mean SampEn values point to visually regular signals such as cyclic functions. Through the proposed methodology, we are able to classify these 23 signals in the unstable category with success because their SampEn is smaller than 0.8 .

4.5. Case 9 Cycle 14 of the Ringhals Stability Benchmark. The data of this case comes from measurements of the Swedish BWR reactor Ringhals 1 [6]. This case consists of a total of 72 LPRMs distributed in two floors or levels of 36 LPRMs each. As stated before, only the analysis of one signal (LPRM 1) is detailed in this work. Figure 24 shows a plot of the studied LPRM 1 signal (of level 4). Figure 25 shows a plot of the studied segment (the red dotted line) that is decomposed through NA-MEMD and its PSD estimate (bottom of figure).

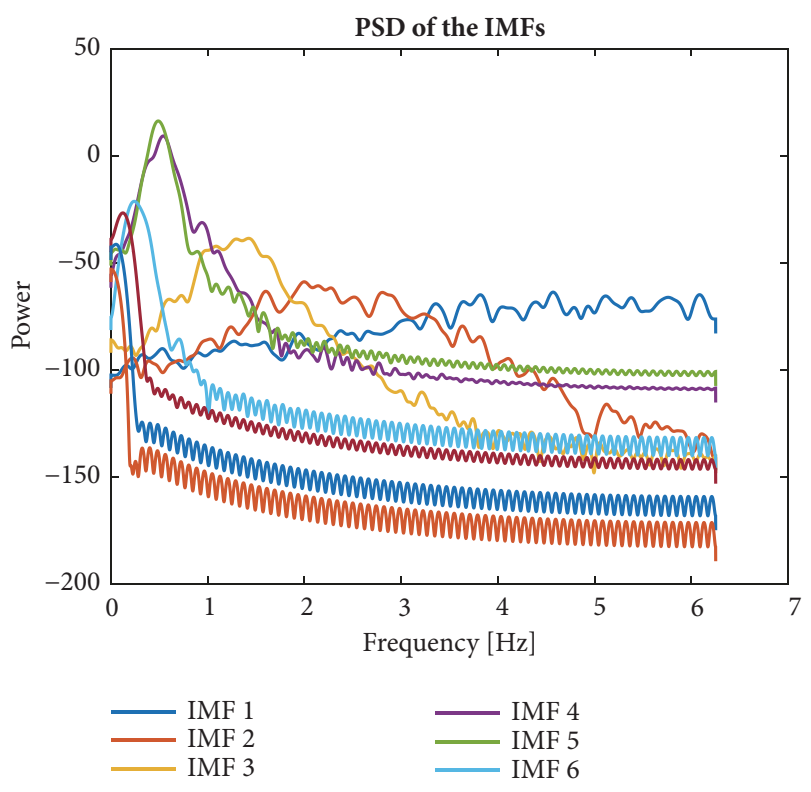

FIGURE 20: PSD estimate of the first 6 IMFs extracted in the analyzed segment.
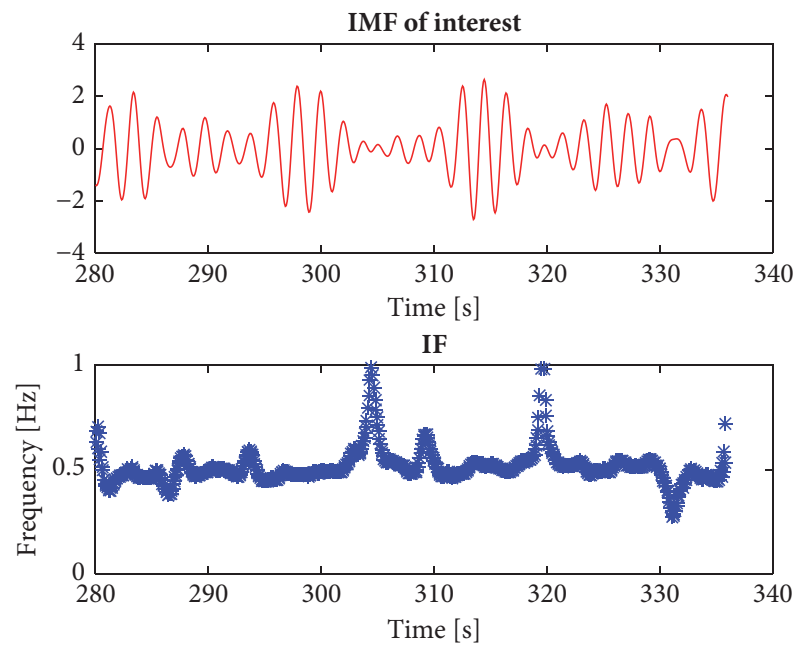

Figure 21: IMF 5 linked to BWR instability and its associated IF (oscillating close to $0.5 \mathrm{~Hz}$ ).

Figure 26 shows the first extracted 5 IMFs (by the way, IMF 4 looks like a cyclic function) of the segment. Figure 27 shows a plot of the PSD estimates of the first 6 IMFs. The PSD estimate of IMF 4 is highly concentrated around $0.5 \mathrm{~Hz}$. Figure 28 shows the tracked IMF 4 of interest linked to BWR instability, this IMF 4 looks like a cyclic function and its associated IF is almost a line centered around $0.5 \mathrm{~Hz}$. The computed SampEn of this IMF 4 is 0.4544 (the studied IMF 4 is unstable. 


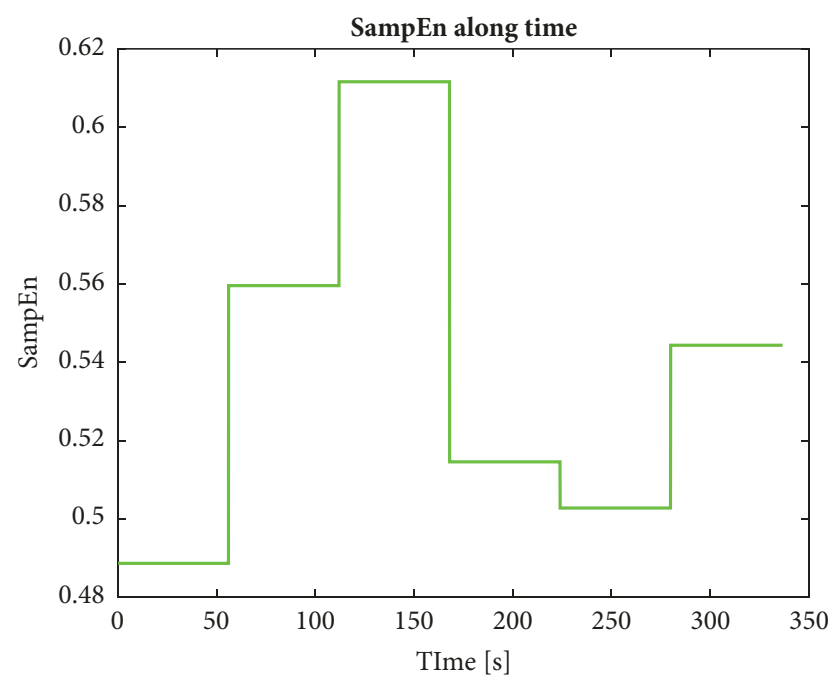

FIGURE 22: SampEn estimates of the IMFs or modes of interest along time.

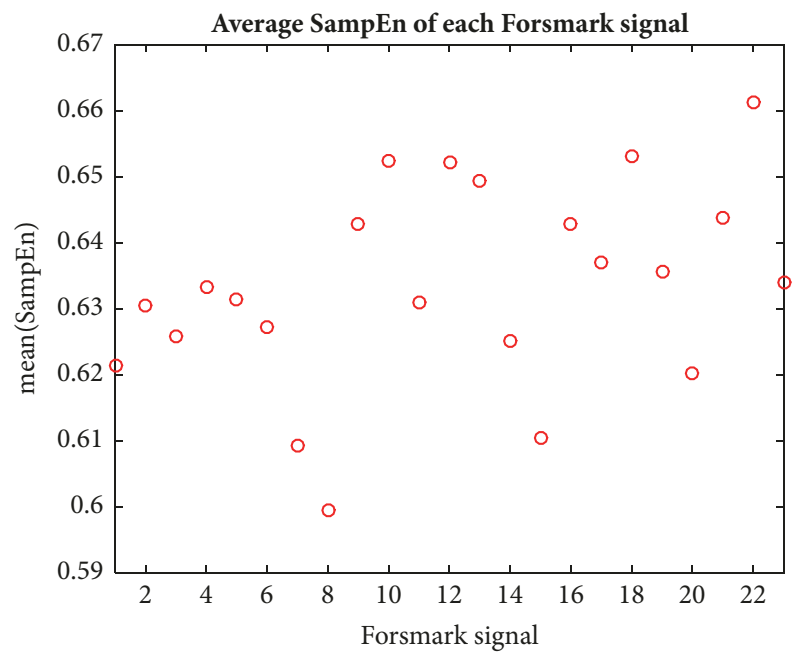

Figure 23: Computed mean(SampEn) of the studied 23 unstable signals (SampEn of the IMFs linked to instability) of the studied Forsmark Case 4.

Thus, the studied segment is unstable). Figure 29 shows the computations of the SampEn along time for all of the studied segments of $60 \mathrm{~s}$. All of the estimates are smaller than 0.8 and located between 0.4-0.6. So, the studied signal is unstable and highly regular like a cyclic function.

Table 4 shows the mean(SampEn), median(SampEn), and std(SampEn) of the time series shown in Figure 29. The mean(IF) and std(IF) of all of the studied segments of the targeted LPRM are also given in this table. Figure 30 shows the computed mean(SampEn) values of the 36 LPRMs of level 2 of the Ringhals reactor. It is observed, that all of the SampEn estimates are smaller than 0.8 (The percentage of classification of the studied signals in the unstable category is of $100 \%)$. The entire floor 2 is unstable and the SampEn estimates are located in the range 0.5-0.7. Figure 31 shows the computed mean(SampEn) values of the 36 LPRMs of

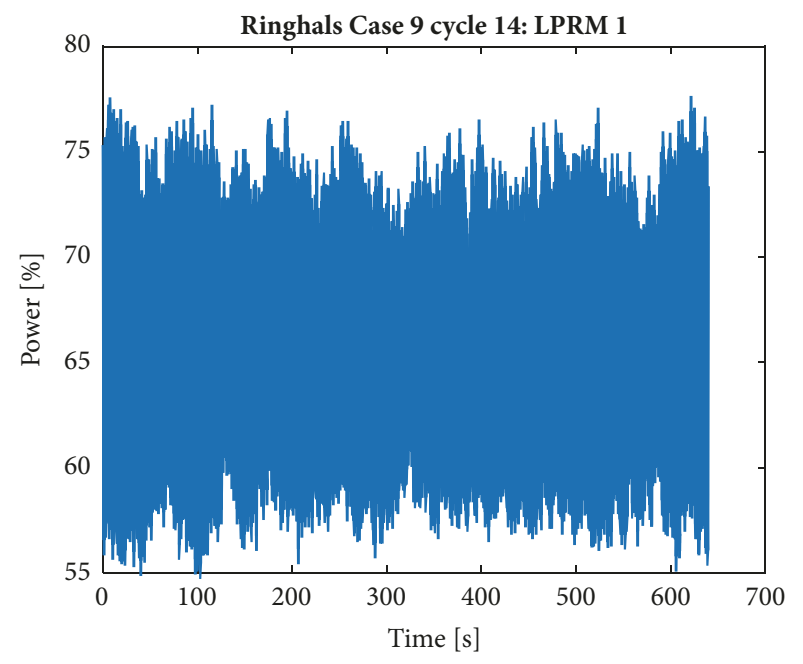

FIgURE 24: Ringhals Case 9 cycle 14 LPRM1.
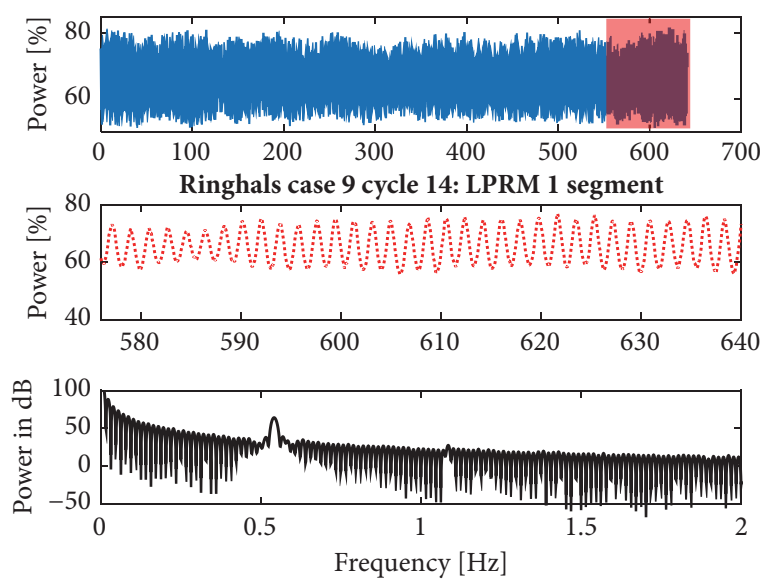

FIGURE 25: Studied segment of the Ringhals Case 9 cycle 14 LPRM 1.
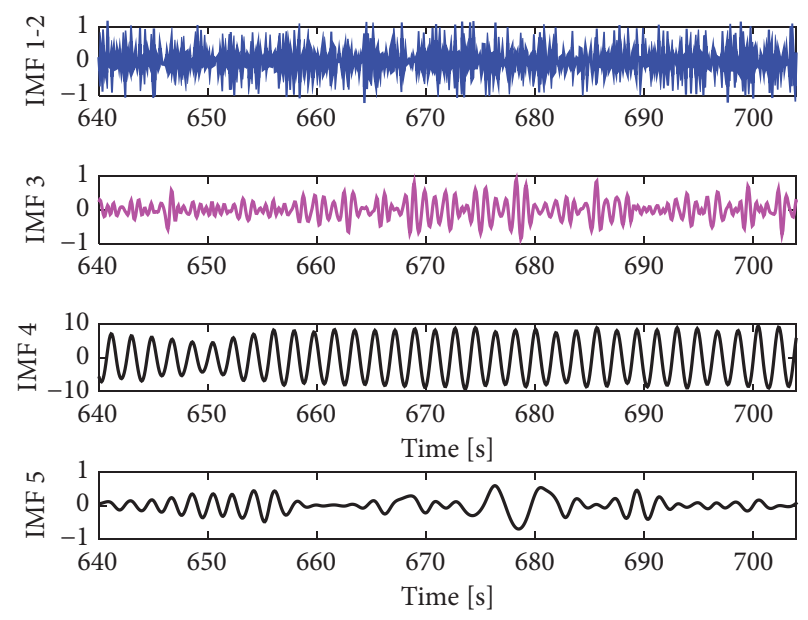

FIGURE 26: A plot of the first 5 IMFs extracted from the analyzed segment of the studied Ringhals case. 
TABle 4: Mean(SampEn), Median(SampEn), and std(Sampen) of the analyzed LPRM 1. Now the mean(IF) and std(IF) are included.

\begin{tabular}{lccccc}
\hline Signal & Mean(SampEn) & Median(SampEn) & Std(SampEn) & $\begin{array}{c}\text { Mean(IF) } \\
{[\mathrm{Hz}]}\end{array}$ & $\begin{array}{c}\text { Std(IF) } \\
{[\mathrm{Hz}]}\end{array}$ \\
\hline Ringhals LPRM 1 & 0.4668 & 0.4730 & 0.0497 & 0.5165 & 0.0328 \\
\hline
\end{tabular}

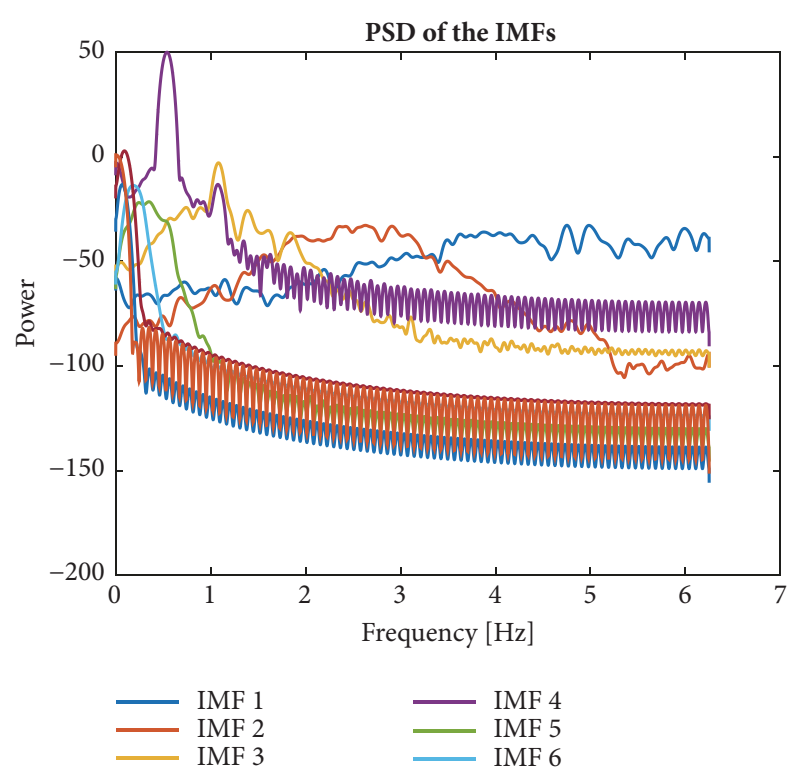

FIGURE 27: PSD estimate of the first 6 IMFs extracted from the analyzed segment.
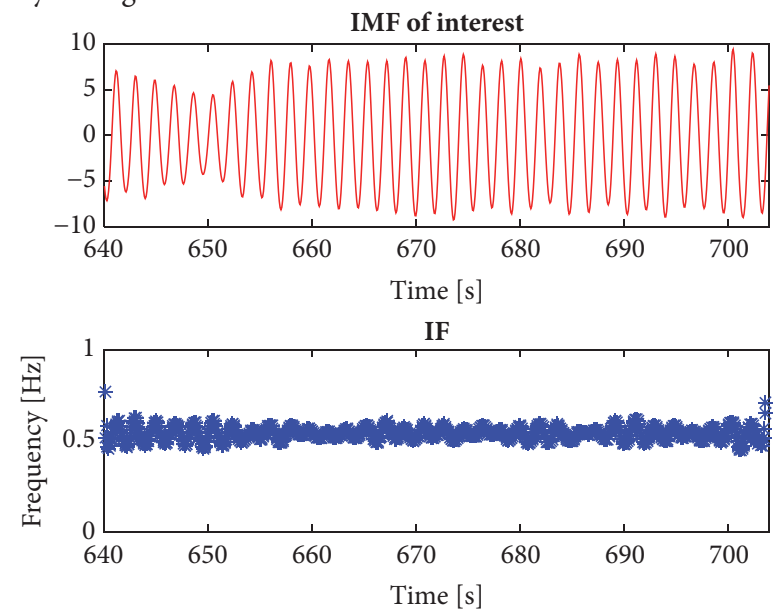

FIGURE 28: IMF 4 linked to BWR instability and its associated IF (very close to $0.5 \mathrm{~Hz}$ ).

level 4 of the Ringhals reactor. It is observed that all of the SampEn estimates are smaller than 0.8 (The percentage of classification of the studied signals in the unstable category is of $100 \%$ as well). The entire floor 4 is also unstable and the SampEn estimates are located in the range 0.45-0.7. The entire case 9 is unstable and SampEn was perfectly able to detect that behavior.

\section{Conclusions}

In this work the Sample Entropy, a measure that provides an index of signal complexity or irregularity of a time series, was

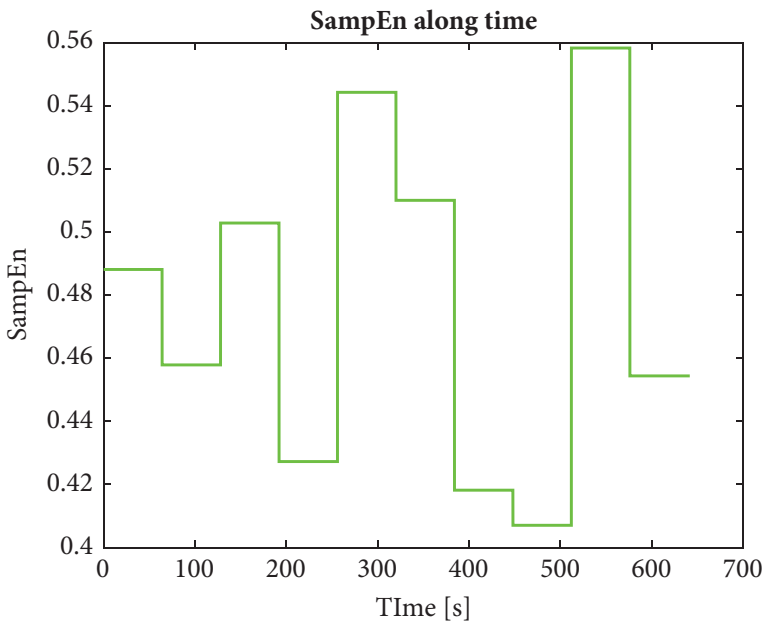

Figure 29: SampEn estimates of the IMFs or modes (IMFs 4) of interest along time.

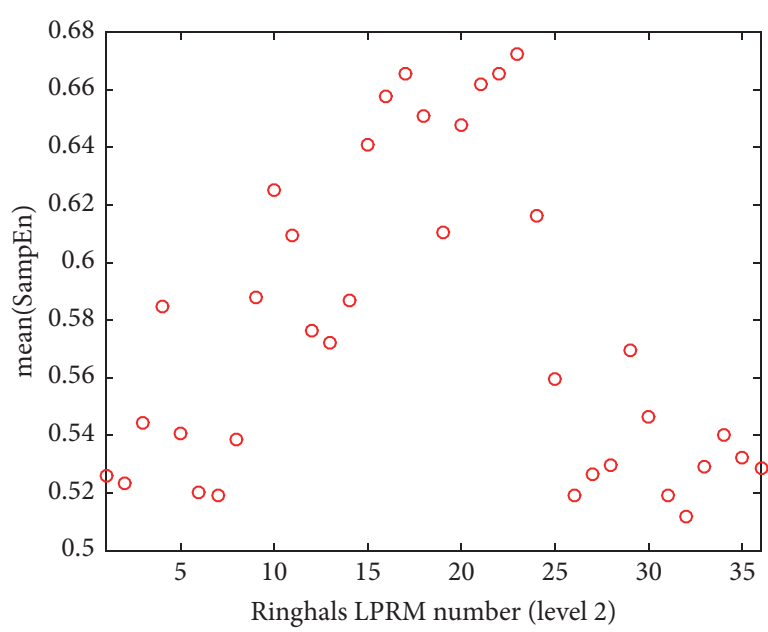

FIGURE 30: Mean(SampEn) values for the 36 LPRMs of level 2 of the Ringhals BWR unit.

used to estimate complexity of a BWR time series for stability analysis. The SampEn was tested in conjunction with the noise-assisted multivariate empirical mode decomposition, a nonlinear filter that decomposes nonstationary data from non-linear sources. The NA-MEMD is used in this application to extract correctly the associated mode to the density wave oscillation, before to compute the SampEn.

The proposed SampEn + NA-MEMD rolling window methodology allowed categorize stable BWR signals from unstable ones. The computed SampEn values of the stable signals oscillated around 1.2 (a high SampEN value is linked to a broad band noncoherent noise (BBNCN) associated with stable behavior). The SampEn values of unstable signals 


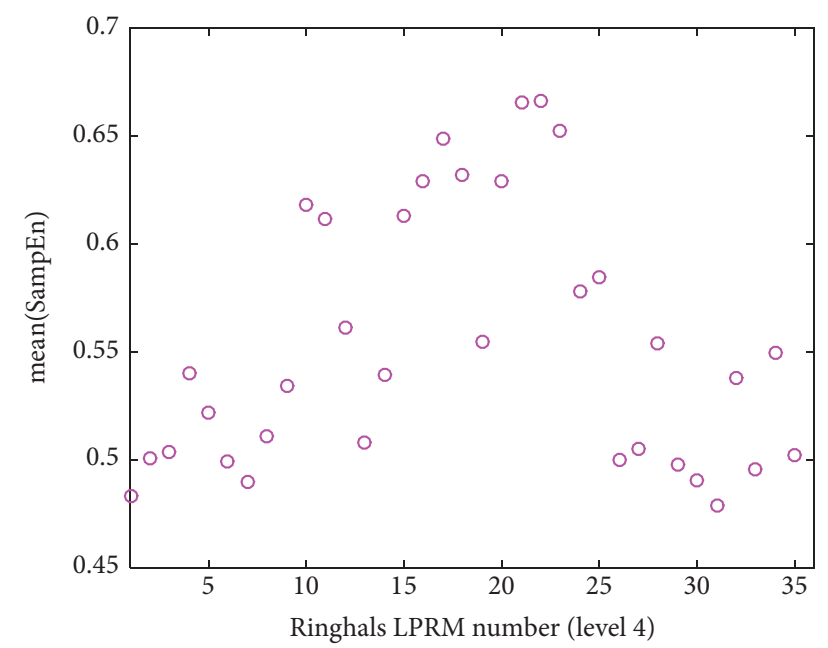

Figure 31: Mean(SampEn) values for the 36 LPRMs of level 4 of the Ringhals BWR unit.

fluctuate close to 0.6 (they might be lower than that, but overall such SampEn estimates are below the threshold SampEn value of 0.8). A low value of SampEn points in this case to a simple cyclic function that is linked to density wave instability. $100 \%$ of the typical BWR studied cases were classified with success into stable or unstable categories. $100 \%$ of the Forsmakr Case 4 studied LPRMs confirm BWR instability through our proposed SampEn + NA-MEMD methodology. $100 \%$ of the studied LPRMs of level 2 and 4 of the Ringhals Case 9 cycle 14 confirm instability. According to these experiments, the SampEn paired with the NAMEMD is a suitable candidate to be used as a BWR stability indicator.

In the future, the SampEn might be tested in conjunction with other suitable nonlinear indicators (possibly fractal dimension estimators [25]) to build a robust BWR stability monitor based on 2 nonlinear measurements, where one of them is going to be the SampEn. In general, the SampEn is a suitable and easy to implement BWR nonlinear stability indicator for real time series analysis.

\section{Nomenclature}

$C_{i}^{m}: \quad$ The embedding vector associated to the embedding dimension $(m)$ in the sample entropy

$C_{i}^{m+1}$ : Defined for dimension $m+1$

$U^{m}:$ An average across all embedding vectors $C_{i}^{m}$

$U^{m+1}$ : An average across all embedding vectors $C_{i}^{m+1}$

$v_{i}$ : A subvector of the studied time series, with $m$ consecutive data points taken from $x$ $\begin{array}{ll}x: & \text { A studied time series } x=\left[\mathrm{x}_{1}, \mathrm{x}_{2}, \ldots, \mathrm{x}_{N}\right] \text { of } \\ & \text { length } N \\ \Theta: & \text { The Heaviside function } \\ \|\cdot\|_{1}: & \text { The Chebyshev distance between } v_{i} \text { and } v_{j}\end{array}$

Acronyms

$\begin{array}{ll}\text { BWR: } & \text { Boiling Water Reactor } \\ \text { DR: } & \text { Decay Ratio } \\ \text { NA-MEMD: } & \text { Noise-assisted multivariate empirical } \\ & \text { mode decomposition } \\ \text { IMF: } & \text { Intrinsic Mode Function } \\ \text { IF: } & \text { Instantaneous Frequency } \\ \text { PSD: } & \text { Power Spectral Density } \\ \text { SampEn: } & \text { Sample Entropy } \\ \text { BBNCN: } & \text { Broad band noncoherent noise } \\ \text { HHT: } & \text { Hilbert-Huang transform. }\end{array}$

\section{Data Availability}

The Forsmark and Ringhals stability benchmark are two different efforts organized by Nuclear Energy Agency (NEA) of the Organisation for Economic Cooperation and Development (OECD) and developed by research laboratories and Nuclear Regulatory Commissions from several countries belonging to the this organisation. The data are available directly with the different participants of this project. The other data used in this article to support the findings of this study were supplied by the Comisión Nacional de Seguridad Nuclear y Salvaguardias (the Mexican Nuclear Regulatory Commission) under license and so cannot be made available.

\section{Conflicts of Interest}

The authors declare that there are no conflicts of interest.

\section{Acknowledgments}

The authors acknowledge the support given by the Mexican Science and Technology National Council (CONACyT) in the form of a Ph.D. scholarship to complete a graduate program at the Autonomous Metropolitan University (UAM) in Mexico City.

\section{References}

[1] H. Ikeda, T. Fukahori, Y. Kubo, H. Soneda, and S. Mizokami, "BWR stability issues in Japan," Science and Technology of Nuclear Installations, vol. 2008, Article ID 358616, 11 pages, 2008.

[2] F. D'Auria, "The BWR stability issue," in THICKET 2008 (Seminar On The Transfer Of Competence, Knowledge And Experience Gained Through CSNI Activities In The Field Of Thermal-Hydraulics), Session IX - Paper 26, 2008.

[3] Y. M. Farawila, D. W. Pruitt, P. E. Smith, L. Sanchez, and L. P. Fuentes, "Analysis of the Laguna Verde instability event," in Proceedings of the National Heat Transfer Conference, vol. 9, pp. 198-202, Houston, USA, 1996. 
[4] W. Wulff, H. S. Cheng, and A. N. Mallen, "Causes of Instability at La Salle and consequences from postulated scram failure," No. BNL-NUREG-45273, CONF-9010224-4, Brookhaven National Lab, Upton, NY, USA.

[5] G. Verdú, D. Ginestar, J. L. Muñoz-Cobo et al., "Forsmark 1\&2 Stability Benchmark. Time series analysis methods for oscillations during BWR operation," Nuclear Science, Final Report, NEA/NSC/DOC(2001)2, New York, USA, 2001.

[6] T. Lefvert, OECD/NEA Ringhals 1 Stability Benchmark, Nuclear Energy Agency, Boulogne-Billancourt, France, 1996.

[7] J. March-Leuba, Density Wave Instabilities in Boiling Water Reactors, Nuclear Regulatory Commission, Div. of Systems Technology; Oak Ridge National Lab., TN, USA, 1992.

[8] C. P. Marcel, Experimental and numerical stability investigations on natural circulation boiling water reactors, IOS press, 2007.

[9] M. Rohde, C. P. Marcel, A. Manera, T. H. J. J. van der Hagen, and B. Shiralkar, "Investigating the ESBWR stability with experimental and numerical tools: a comparative study," Nuclear Engineering and Design, vol. 240, no. 2, pp. 375-384, 2010.

[10] T. H. J. J. Van Der Hagen, R. Zboray, and W. J. M. De Kruijf, "Questioning the use of the decay ratio in BWR stability monitoring," Annals of Nuclear Energy, vol. 27, no. 8, pp. 727732, 2000.

[11] I. Pázsit, "Determination of reactor stability in case of dual oscillations," Annals of Nuclear Energy, vol. 22, no. 6, pp. 377387, 1995.

[12] E. Gialdi, S. Grifoni, C. Parmeggiani, and C. Tricoli, "Core stability in operating BWR: operational experience," Progress in Nuclear Energy, vol. 15, no. C, pp. 447-459, 1985.

[13] J. S. Richman and J. R. Moorman, "Physiological time-series analysis using approximate entropy and sample entropy," American Journal of Physiology-Heart and Circulatory Physiology, vol. 278, no. 6, pp. H2039-H2049, 2000.

[14] S. Li, Q. Zhou, S. Wu, and E. Dai, "Measurement of climate complexity using sample entropy," International Journal of Climatology, vol. 26, no. 15, pp. 2131-2139, 2006.

[15] Y. Jia, H. Gu, and Q. Luo, "Sample entropy reveals an agerelated reduction in the complexity of dynamic brain," Scientific Reports, vol. 7, no. 1, 2017.

[16] N. Rehman and D. P. Mandic, "Multivariate empirical mode decomposition," Proceedings of the Royal Society A: Mathematical, Physical and Engineering Sciences, vol. 466, pp. 1291-1302, 2010.

[17] Z. H. Wu and N. E. Huang, "Ensemble empirical mode decomposition: a noise-assisted data analysis method," Advances in Adaptive Data Analysis (AADA), vol. 1, no. 1, pp. 1-41, 2009.

[18] N. E. Huang, Z. Shen, S. R. Long et al., "The empirical mode decomposition and the Hilbert spectrum for nonlinear and non-stationary time series analysis," Proceedings of the Royal Society Series A, vol. 454, pp. 903-995, 1998.

[19] O. A. Olvera-Guerrero, A. Prieto-Guerrero, and G. EspinosaParedes, "Non-linear boiling water reactor stability with Shannon Entropy," Annals of Nuclear Energy, vol. 108, pp. 1-9, 2017.

[20] O. A. Olvera-Guerrero, A. Prieto-Guerrero, and G. EspinosaParedes, "Non-linear stability analysis of real signals from nuclear power plants (Boiling Water Reactors) based on noise assisted empirical mode decomposition variants and the Shannon Entropy," Entropy, vol. 19, no. 7, Article ID 359, 2017.

[21] C. E. Shannon, "A mathematical theory of communication," Bell System Technical Journal, vol. 27, pp. 379-423, 1948.
[22] K. Chon, C. Scully, and S. Lu, "Approximate entropy for all signals," IEEE Engineering in Medicine and Biology Magazine, vol. 28, no. 6, pp. 18-23, 2009.

[23] M. B. Kennel, R. Brown, and H. D. I. Abarbanel, "Determining embedding dimension for phase-space reconstruction using a geometrical construction," Physical Review A: Atomic, Molecular and Optical Physics, vol. 45, no. 6, pp. 3403-3411, 1992.

[24] N. Rehman and D. P. Mandic, "Filter bank property of multivariate empirical mode decomposition," IEEE Transactions on Signal Processing, vol. 59, no. 5, pp. 2421-2426, 2011.

[25] C. Demazière, C. Marcel, M. Rohde, and T. Van Der Hagen, "Multifractal analysis of chaotic flashing-induced instabilities in boiling channels in the natural-circulation CIRCUS facility," Nuclear Science and Engineering, vol. 158, no. 2, pp. 164-193, 2008. 

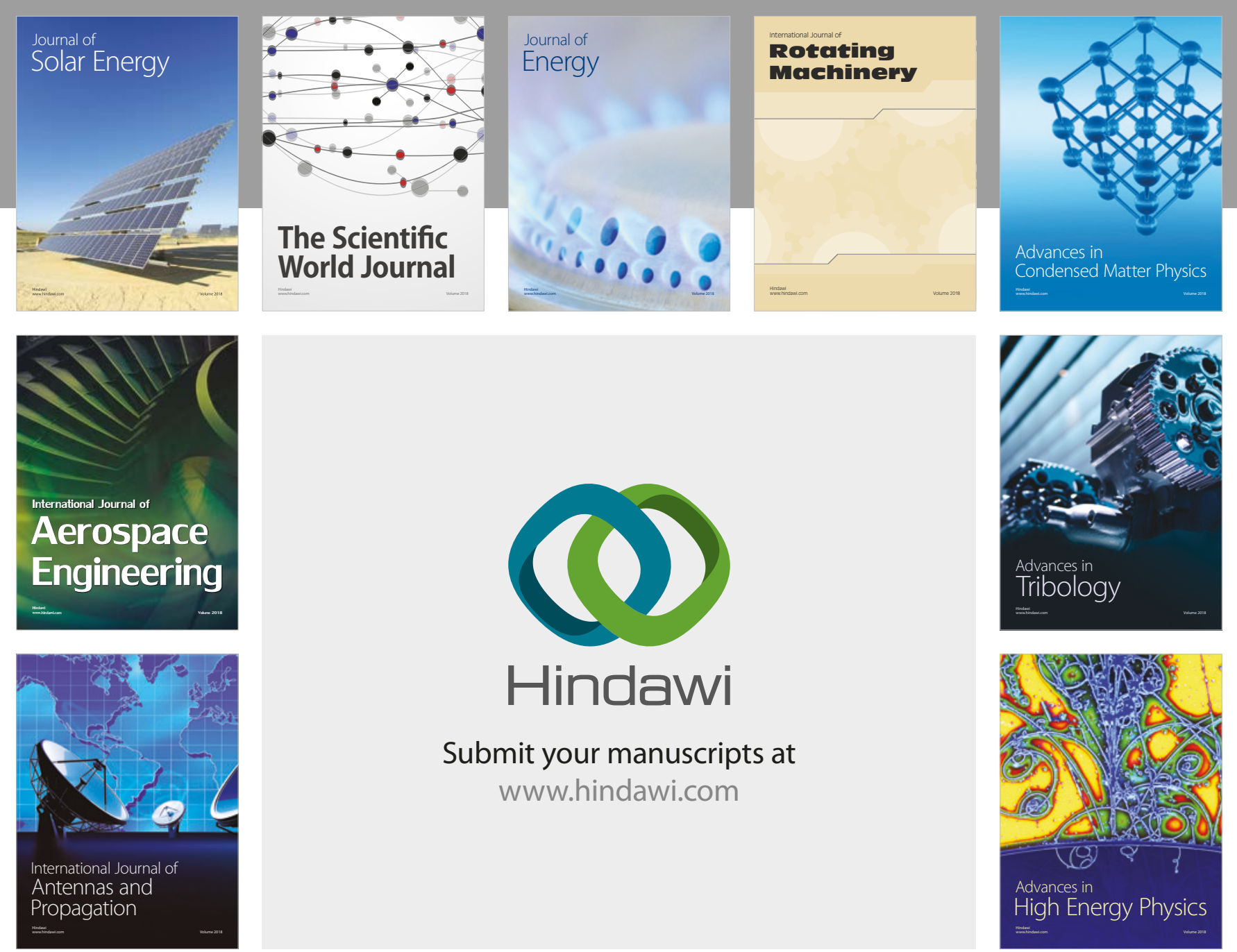

Submit your manuscripts at

www.hindawi.com
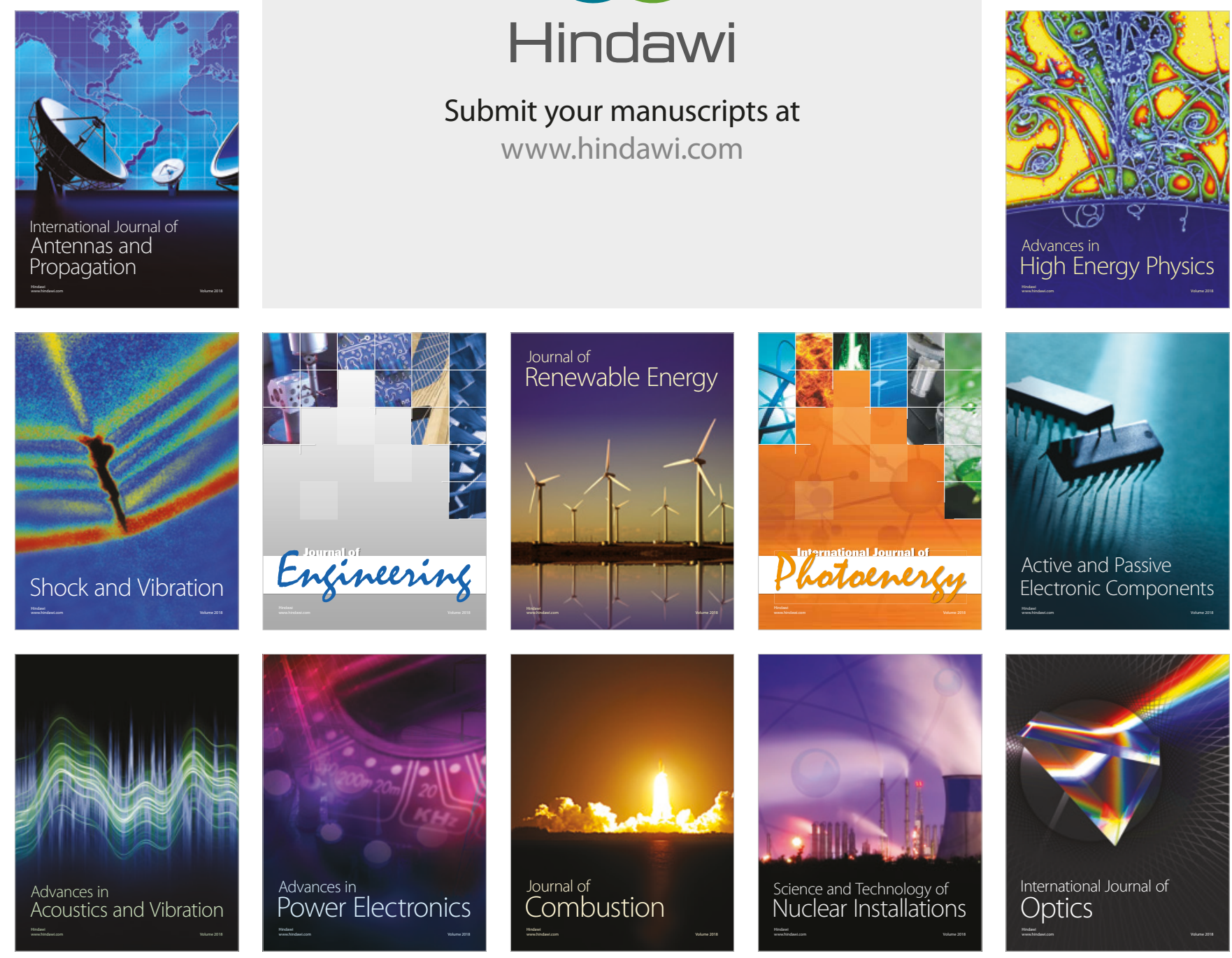